\title{
The Beneficial Effects of Valproic Acid in Thyroid Cancer Are Mediated through Promoting Redifferentiation and Reducing Stemness Level: An In Vitro Study
}

\author{
Vahid Haghpanah, ${ }^{1}$ Mohsen Malehmir, ${ }^{2,3}$ Bagher Larijani, ${ }^{1}$ Shahin Ahmadian, ${ }^{2}$ \\ Kamran Alimoghaddam, ${ }^{3}$ Ramin Heshmat, ${ }^{1}$ \\ Ardeshir Ghavamzadeh, ${ }^{3}$ Khadijeh Adabi, ${ }^{1}$ and Seyed H. Ghaffari ${ }^{3}$ \\ ${ }^{1}$ Endocrinology and Metabolism Research Center, Endocrinology and Metabolism Clinical Sciences Institute, \\ Tehran University of Medical Sciences, P.O. Box 1411413137, Tehran, Iran \\ ${ }^{2}$ Institute of Biochemistry and Biophysics (IBB), University of Tehran, P.O. Box 13145-1384, Tehran, Iran \\ ${ }^{3}$ Hematology, Oncology, and Bone Marrow Transplantation Research Center, Tehran University of Medical Sciences, \\ P.O. Box 1411413137, Tehran, Iran
}

Correspondence should be addressed to Seyed H. Ghaffari; shghaffari200@yahoo.com

Received 22 September 2013; Revised 11 April 2014; Accepted 28 April 2014; Published 15 May 2014

Academic Editor: Thomas J. Fahey

Copyright (C) 2014 Vahid Haghpanah et al. This is an open access article distributed under the Creative Commons Attribution License, which permits unrestricted use, distribution, and reproduction in any medium, provided the original work is properly cited.

\begin{abstract}
Valproic acid (VPA) has been identified as a histone deacetylase inhibitor, inducing differentiation in transformed cells. However, no study has shown the effect of VPA in the redifferentiation induction and stemness of anaplastic thyroid. The main objective of this study was to evaluate the efficacy of VPA as a differentiation therapy agent in human thyroid cancer based on its effect on stemness and differentiation process. Indications for differentiation of 8305C and B-CPAP cell lines following VPA treatment were obtained by analyzing cell proliferation rate, morphological changes, adherent-dependent colony formation, and Hoechst 33342 staining. The expressions of stemness, differentiation, and aggressiveness specific marker genes were measured by quantitative RT-PCR. VPA treatment effectively showed growth inhibition in both cell lines. The high nuclear-cytoplasmic $(\mathrm{N}: \mathrm{C})$ ratio of $8305 \mathrm{C}$ cells markedly decreased and treated cells became more epithelial-like. Treated cells showed stronger Hoechst 33342 fluorescence compared with control cells. The hTERT and OCT-4 reduction was paralleled with adherent-dependent colony formation decrement in both cell lines. VPA effectively induced NIS and TTF-1 in anaplastic cells, it whereas showed no clear pattern in papillary cell line. VPA treatment also resulted in the reduction of MMP-2 and MMP-9. These finding suggest that VPA could redifferentiate the anaplastic thyroid cancer cells.
\end{abstract}

\section{Introduction}

Treatment of advanced thyroid cancer with follicular cell origin, mainly radioactive resistant papillary thyroid cancer (PTC), and iodine/chemotherapy resistant anaplastic thyroid carcinoma (ATC) is notoriously challenging $[1,2]$. This is partly because these malignancies have lost the ability to take up radioactive iodine or have dedifferentiated to more aggressive types, making treatments ineffective [2]. According to multistep and fetal cell carcinogenesis models, thyroid carcinoma originates from well-differentiated normal thyroid follicular cells [3], as a consequence of multiple mutations accumulated throughout the entire life span, or as a remnant of fetal thyroid cells [4]. However, recent work has argued that thyroid cancer, parallel to other solid tumors, might originate from thyroid cancer stem cells [5-7].

Thyroid cancer stem-like or stem cells are undifferentiated cells which do not express NIS and thus cannot absorb radioactive iodine by the same manner that welldifferentiated thyroid cells do. Conventional modalities for thyroid cancer, surgery, radiotherapy, and chemotherapy do not appear to be effective in the targeting tumor-initiating 
cells; therefore, an alternative therapeutic plan targeting stem-like or stem cells is required to effectively target cancer stem cells. Differentiation therapy which includes methods and therapeutic strategies aiming to force the cancer cells to resume the process of maturation seems promising [8].

Histone deacetylase (HDAC) inhibitors have recently been an object of interest to clinicians for their potential use in cancer therapy as antiproliferative agents exerting their influence by inducing apoptosis, promoting differentiation, and arresting cell cycle [9]. VPA, an established drug in the long-term therapy of epilepsy, has been suggested as a potential agent for differentiation of mesenchymal stem cells [10] and a number of tumors such as glioblastoma [11], head and neck cancer [12], and uveal melanoma [13].

Here, to attribute the redifferentiation and aggressiveness reduction responsible for therapeutic role of VPA in thyroid carcinoma cell lines, we treated ATC (8305C) and PTC (BCPAP) cell lines with various doses of VPA and monitored the responses by means of cell proliferation, $\mathrm{N}$ : $\mathrm{C}$ ratio and morphological analysis, adherent-dependent colony formation assay, quantitative RT-PCR, and Hoechst 33342 staining.

\section{Materials and Methods}

2.1. Cell Lines and VPA Treatment. Two human thyroid carcinoma cell lines used in this study, B-CPAP and 8305C, were purchased from the DSMZ (Braunschweig, Germany). These cell lines were cultured, as previously described [14]. VPA (Sigma-Aldrich) was diluted at desired concentration in the cell culture medium and treated with $0.1,1,5$, and $10 \mathrm{mmol} / \mathrm{L}$. Equal volume of DMSO was added to the control samples, in which the final concentration of DMSO did not exceed more than $0.1 \%$ of the final medium.

2.2. Proliferation Assay (MTT). Cell proliferation was performed to calculate the appropriate dose for further assays. Briefly, 5000 cells were cultured in a 96-well plate and treated for $96 \mathrm{~h}$ with VPA. Then, the MTT solution was added with final concentration of $5 \mathrm{mg} / \mathrm{mL}$. Medium was aspirated after $4 \mathrm{~h}$ of incubation, and to solubilize the formazan crystals $100 \mu \mathrm{L}$ DMSO was added. VPA growth inhibition rate was calculated using the following equation: inhibition rate (\%) = optical density $(\mathrm{OD})_{\text {obs }}$ /optical density $(\mathrm{OD})_{\text {con }} \times 100$ in which $\mathrm{OD}_{\mathrm{obs}}$ and $\mathrm{OD}_{\text {con }}$ represent the optical densitometry of treated and untreated control (DMSO) cells, respectively.

2.3. N:C Ratio and Morphological Analysis. The $8305 \mathrm{C}$ cell line was cultured in a 6-well plate and was treated the day after. Briefly, cultured cells were treated with 0.1 and $1 \mathrm{mmol} / \mathrm{L}$ of VPA for $96 \mathrm{~h}$ and were fixed in $0.1 \mathrm{M}$ phosphate buffered saline (PBS), $2.5 \%$ glutaraldehyde ( $\mathrm{pH} 7.4$ ). The $\mathrm{N}$ : C ratio and morphological studies were performed under light microscope after Wright-Giemsa (Sigma-Aldrich, Germany) staining, according to the manufacturer instructions. The stained blue to violet area was defined as cytoplasm; in contrast, the stained red to pink area was defined as nucleus. Image J software (NIH Image, Bethesda, MD) was used to analyze the $\mathrm{N}: \mathrm{C}$ ratio changes. The $\mathrm{N}: \mathrm{C}$ ratio was derived from the $\mathrm{N}: \mathrm{C}$ ratio $=\mathrm{Ave}_{\text {nuc }} / \mathrm{Ave}_{\text {cyt }} \times 100$ relationship, in which Ave $e_{\text {nuc }}$ and Ave $e_{\text {cyt }}$ show mean areas of the nucleus and cytoplasm. Stained cells were studied for morphological changes with light microscopy $(\times 40)$.

2.4. Adherent-Dependent Colony Formation. To evaluate the colony formation ability of the $8305 \mathrm{C}$ and B-CPAP cells following 0.1 and $1 \mathrm{mmol} / \mathrm{L}$ VPA treatment, adherent-dependent colony formation assay was carried out. The colony-forming efficiency (CFE) of each cell line was obtained as CFE (\%) = number of colonies/initial seeding density $\times 100$. The data represent the number of colony forming cells in each well after initial seeding.

2.5. RNA Extraction and Quantitative Real-Time PCR. Total RNA was extracted from VPA treated cell lines by using High Pure RNA Isolation Kit (Roche, Indianapolis, IN, USA). RNA $(1 \mu \mathrm{g})$ was reverse transcribed with Prime Script RT reagent kit (Takara), using random hexamer and oligo(dT) primers. Expression of mRNAs was measured by quantitative real-time PCR using StepOnePlus (Applied Biosystem, USA) instrument using SYBER green PrecisionTM 2X qPCRMastermix (PrimerDesign Ltd., UK). Reaction mixture included the following: SYBER green master mix $(10 \mu \mathrm{L}), \mathrm{cDNA}(2 \mu \mathrm{L})$, forward and reverse primers (10 picomol), and nuclease free water $(7 \mu \mathrm{L})$ which were added in a final volume of $20 \mu \mathrm{L}$. Thermocycling included a single initial heat inactivation and denaturation incubation at $95^{\circ} \mathrm{C}$ for 10 minutes, followed by 40 cycles of $95^{\circ} \mathrm{C}$ for $5 \mathrm{~s}$ and a combined annealing/extension step for $30 \mathrm{~s}$ at $60^{\circ} \mathrm{C}$. Hypoxanthine phosphoribosyltransferase 1 (HPRT) was amplified as normalizer, and relative-fold differences of target were calculated using the $2^{-\Delta \Delta C t}$ method normalized to HPRT levels. Primers sequences are indicated in Table 1.

2.6. Hoechst 33342 Staining. The dilution of single 8305C cells was cultured on the surface of the glass coverslip in 6-well plates and treated with 0.1 and $1 \mathrm{mmol} / \mathrm{L}$ of VPA for $96 \mathrm{~h}$. The medium was aspirated and cells were washed twice with PBS pH 7.4 solution. In order to permeabilize the cells, cold $\left(-20^{\circ} \mathrm{C}\right) 100 \%$ methanol was added and left for 15 minutes in room temperature and rinsed thoroughly three times with PBS. Fresh dilution of the Hoechst 33342 stock solution was added with the final concentration of $1 \mu \mathrm{g} / \mathrm{mL}$, incubated at $37^{\circ} \mathrm{C}$ for 15 minutes, and protected from light. The stained cells were immediately examined by IX70 fluorescence microscope (Olympus Optical, London, UK) using blue filter and photographed (excitation, $350 \mathrm{~nm}$; emission, $450 \mathrm{~nm}$ ).

2.7. Statistical Analysis. Experimental data are expressed by mean \pm standard deviation of three independent assays for MTT, qRT-PCR, and duplicate for colony formation assay for both cell lines. An independent $t$-test was conducted for comparison between doses. Statistical significance was calculated using paired two-tailed Student's $t$-tests. Statistically different values were defined as significant at ${ }^{*} P<0.05,{ }^{*} P<0.01$, ${ }^{* * *} P<0.001$. Statistically different values for comparison 
TABLE 1: Primer sequences for quantitative RT-PCR.

\begin{tabular}{lcccc}
\hline Gene & Accession number & Forward primer $\left(5^{\prime}-3^{\prime}\right)$ & Reverse primer $\left(5^{\prime}-3^{\prime}\right)$ & Size $(\mathrm{bp})$ \\
\hline HPRT & NM_000194 & TGGACAGGACTGAACGTCTTG & CCAGCAGGTCAGCAAAGAATTTA & 111 \\
NIS & NM_000453 & TGCGGGACTTTGCAGTACATT & TGCAGATAATTCCGGTGGACA \\
TTF-1 & NM_001079668 & AGCACACGACTCCGTTCTC & GCCCACTTTCTTGTAGCTTTCC & 133 \\
p21 & NM_000389 & CCTGTCACTGTCTTGTACCCT & GCGTTTGGAGTGGTAGAAATCT & 130 \\
OCT-4 & NM_001173531 & CTTGAATCCCGAATGGAAAGGG & GTGTATATCCCAGGGTGATCCTC \\
hTERT & NM_001193376 & AACCTTCCTCAGCTATGCCC & GCGTGAAACCTGTACGCCT \\
PAX-8 & NM_003466 & TGGGGACTACAAACGCCAGA & GCTGTCCATAGGGAGGTTGAAT & 210 \\
c-MYC & NM_002467 & CCACAGCAAACCTCCTCACAG & GCAGGATAGTCCTTCCGAGTG \\
MMP-2 & NM_004530 & CTTCCAAGTCTGGAGCGATGT & TACCGTCAAAGGGGTATCCAT & 105 \\
MMP-9 & NM_004994 & GGGACGCAGACATCGTCATC & TCGTCATCGTCGAAATGGGC & 139 \\
\hline
\end{tabular}

HPRT: Hypoxanthine phosphoribosyltransferase 1; NIS: sodium/iodide symporter; TTF1: thyroid transcription factor 1; p21: CIP1/WAF1; OCT-4: octamerbinding transcription factor 4; hTERT: human telomerase reverse transcriptase; PAX8: Paired box gene 8; c-MYC: v-myc myelocytomatosis viral oncogene homolog; MMP-2: matrix metallopeptidase 2; MMP-9: matrix metallopeptidase 9.

between $8305 \mathrm{C}$ and B-CPAP cells were defined as significant at ${ }^{+} P<0.05,{ }^{++} P<0.01,{ }^{+++} P<0.001$.

\section{Results}

3.1. Effect of VPA on Cell Growth. In this study we used MTT assay to determine the cell growth inhibition and also calculate the appropriate doses for the next assays. As shown in Figure 1, VPA treatment showed a 2\% to 52\% inhibition in the $8305 \mathrm{C}$ cells and $2 \%$ to $92 \%$ in the B-CPAP cells. Since we aimed to evaluate the redifferentiation effect of VPA low doses, 0.1 and $1 \mathrm{mmol} / \mathrm{L}$ which are therapeutically achievable in patient, these appropriate doses with low proliferation inhibition were selected for the next assays.

3.2. Significant Morphological Changes Accompanied with N: C Ratio Decrement. N : C ratio measurement was carried out to investigate the beneficial effect of VPA in redifferentiation induction of $8305 \mathrm{C}$ cells. The morphology of Wright-Giemsa stained cells showed that the control cells had spindle shape morphology (Figure 2(a)). In contrast, 0.1 and $1 \mathrm{mmol} / \mathrm{L} \mathrm{VPA}$ treated cells (Figures 2(b) and 2(c)) were ovalround to polygonal. The results of the study revealed that high $\mathrm{N}$ : C ratio of $8305 \mathrm{C}$ cells reduced following VPA treatment. As shown in Figure 2(d), the $\mathrm{N}: \mathrm{C}$ ratio of the cells reduced to $77 \%(P<0.0001)$ and $67 \%(P<0.0001)$ compared to the untreated control (DMSO) in 0.1 and $1 \mathrm{mmol} / \mathrm{L}$ treated cells, respectively. In addition, the cells became larger following VPA treatment. Furthermore, mesenchymal morphology of the cells became more epithelial-like after treatment.

3.3. VPA Suppressed the Colony Forming Capacity. To evaluate the number of the colony forming cells, mainly attributed to progenitor or stem cells, we performed anchoragedependent colony forming assay. As shown in Figure 3, VPA reduced the colony forming ability of the two cell lines. CFE in 6-well plate in the absence of VPA ranged from $2.7 \%$ for BCPAP cells to $3.6 \%$ for $8305 \mathrm{C}$ cells. VPA at 0.1 and $1 \mathrm{mmol} / \mathrm{L}$ exhibited $25 \%$ and $89 \%$ inhibition of anchorage-dependent growth in $8305 \mathrm{C}$ and also $4 \%$ and $89 \%$ in B-CPAP cell line,

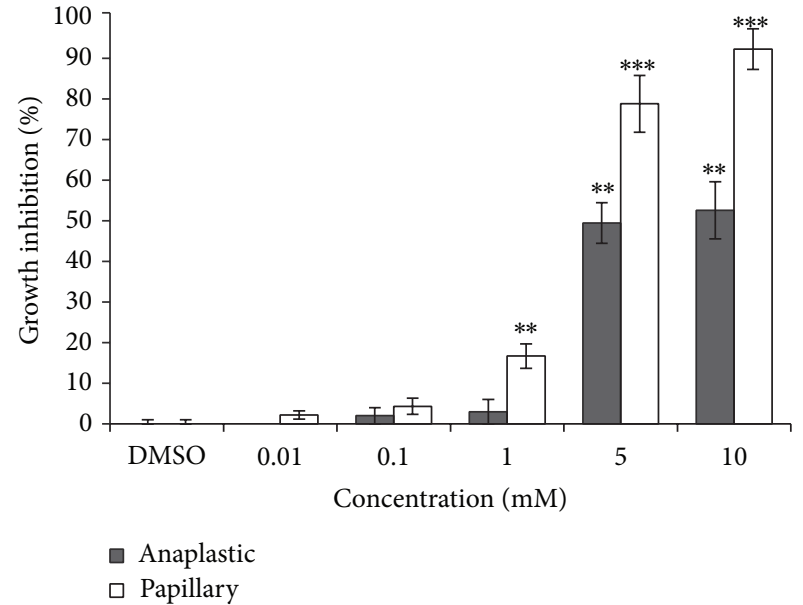

Figure 1: Effects of $0.01,0.1,1,5$, and $10 \mathrm{mmol} / \mathrm{L}$ VPA treatment on $8305 \mathrm{C}$ and B-CPAP cell growth inhibition (GI). Proliferation was measured with an MTT test after $96 \mathrm{~h}$ of proliferation in the presence of appropriate amount of DMSO as control or desired VPA. Data are presented as mean of three experiments \pm SD. Statistical significance was defined at ${ }^{*} P<0.05,{ }^{* *} P<0.01,{ }^{* * *} P<0.001$ compared to the corresponding control.

respectively. These results demonstrated that VPA was able to hinder the anchorage-dependent growth of $8305 \mathrm{C}$ and $\mathrm{B}$ CPAP cells, indicating a persistent downregulation of growth in the presence of VPA.

3.4. VPA-Induced Thyroid Differentiation Marker Accompanied with Downregulation of Stemness and Invasion Related Genes. In this study we evaluated the effect of VPA on the expression pattern of stem cell markers, OCT-4 and hTERT; thyroid-specific differentiation markers, NIS, TTF1, and PAX8; invasion related markers, MMP2 and MMP9, as categorized in Table 2, by using quantitative real-time PCR. VPA treatment, as shown in Figure 4, decreased the expression of OCT-4 and hTERT in the 8305C (Figure 4(a)) and B-CPAP (Figure 4(b)) cells, with marked reduction in the 


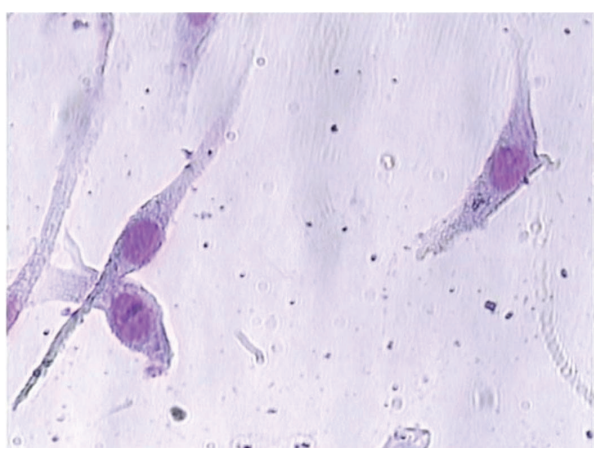

(a)

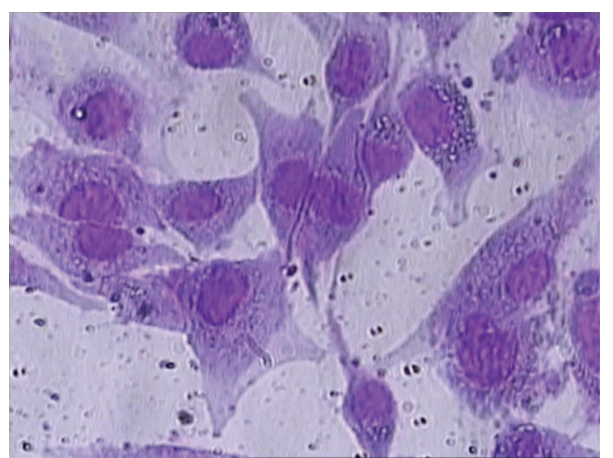

(c)

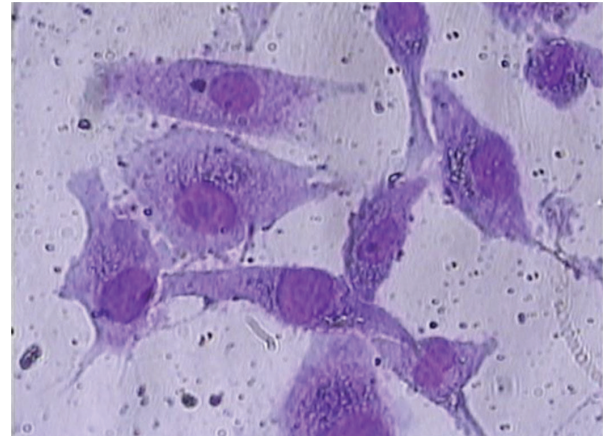

(b)

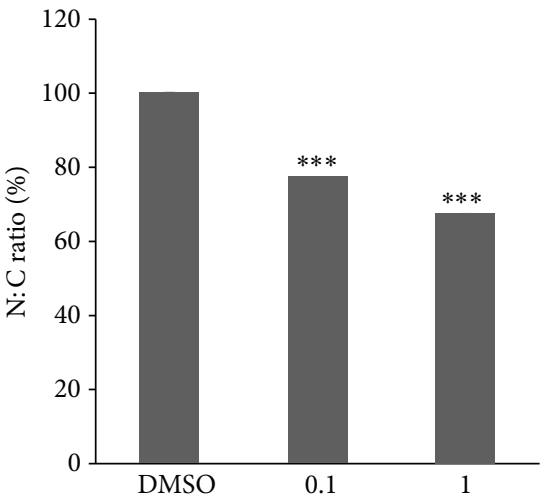

(d)

Figure 2: Morphological changes and N : C ratio analysis of 8305C cells following 0.1 and $1 \mathrm{mmol} / \mathrm{L}$ VPA treatment for $96 \mathrm{~h}$. (a) Untreated control cells (DMSO) showed spindle and mesenchymal morphology. (b) and (c) 0.1 and $1 \mathrm{mmol} / \mathrm{L}$ VPA treated cells showed oval-round to polygonal morphology (magnification 40x). (d) High N:C ratio of the untreated cells decreased dramatically in VPA treated cells. Data are shown as mean $\pm \mathrm{SD}$. ${ }^{* * *} P<0.001$.

8305C cells. The downregulation of $c-M Y C$, the main positive regulator of the $h T E R T$, correlated with the expression of hTERT in the $8305 \mathrm{C}$ cells. The expression of thyroid-specific differentiation markers NIS, TTF1, and PAX8 significantly induced in the $8305 \mathrm{C}$ cell lines. The B-CPAP, however, showed no changes in NIS and decreases in TTF1 expression. The invasive related MMP2 and MMP9 gene expression was reduced in the $8305 \mathrm{C}$ cell line in a dose-dependent manner, and also MMP2 expression was reduced in the B-CPAP cell line. Although MMP9 expression was reduced in low doses in B-CPAP cell line, increased expression was observed in the highest concentration.

\subsection{Brighter Hoechst 33342 Blue Fluorescence in VPA-Treated} Cells. To determine whether aforementioned observations following VPA treatment are pertinent to the stemness of anaplastic cells, that is, whether redifferentiation affects the Hoechst 33342 accumulation attributed to stem-like characteristics, we analyzed the fluorescence microscopy photographs following VPA treatment. As shown in Figure 5(a), 8305C control cells demonstrated relatively weak and dull staining with Hoechst 33342 . In contrast, 0.1 and $1 \mathrm{mmol} / \mathrm{L}$ VPA treated cells showed a strongly bright and homogenous blue fluorescence (Figures 5(b) and 5(c)). The weak staining of control cells might be explained by high expression of efflux transporter in stem cells, a key property of side population cells, whereas VPA treated cells have lost the efflux ability following redifferentiation.

\section{Discussion}

Different carcinogenesis models have been proposed to describe the cellular origin of thyroid cancer. According to multistep and fetal cell carcinogenesis models, thyroid carcinoma originates from well-differentiated normal thyroid follicular cells [3], or remnant of fetal thyroid cells [4]. Recently, however, research findings support the concept that a small population of thyroid cancer cells displays properties characteristic of stem cells [15-17]. These putative cancer-forming entities are able to drive tumorigenesis; they might also mediate metastasis and are resistant to the effects of chemotherapy and radiation therapy [17]. The resistance of stem cells has important implications for current therapeutic approaches which mainly target the rapidly dividing cells. The implication of the resistance for conventional treatment modalities is that there should be new therapeutic strategies to eradicate tumor initiating subpopulation. Currently, few options are available to treat advanced thyroid cancers, which tend 


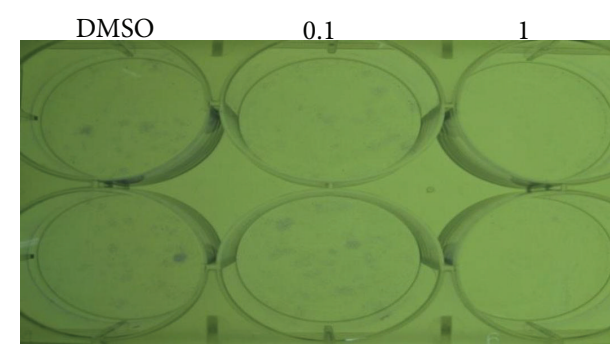

(a)

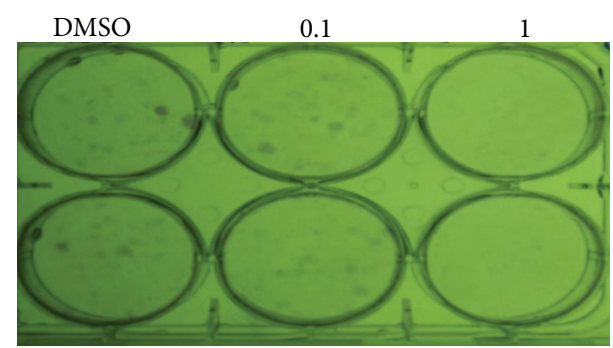

(b)

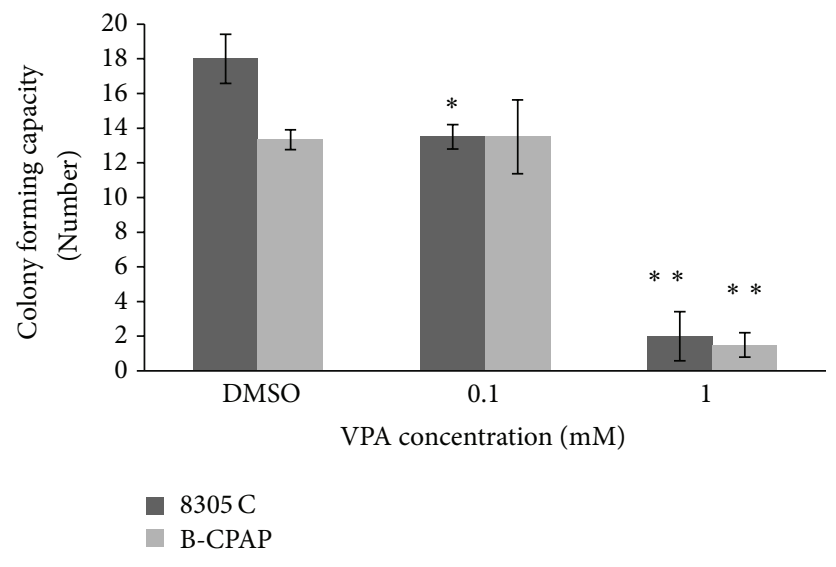

(c)

FIGURE 3: Colony forming capacity of the cells after VPA treatment. Papillary (a) and anaplastic (b) cells were seeded in 6-well plate, and after attachment for $3 \mathrm{~h}$, cells were treated with 0.1 and $1 \mathrm{mmol} / \mathrm{L}$ of VPA for 2 weeks. After 2 weeks colonies were fixed with glutaraldehyde (6.0\%, $\mathrm{v} / \mathrm{v})$, stained with crystal violet $(0.5 \% \mathrm{w} / \mathrm{v})$ and the number of colonies with at least 50 cells was counted. (c) Each data is expressed as mean of colony forming cells \pm SD. Statistically different values of ${ }^{*} P<0.05,{ }^{* *} P<0.01$, and ${ }^{* * *} P<0.001$ were determined significantly compared with the corresponding control.

TABLE 2: List of genes that were used and categorized based on their biological function.

\begin{tabular}{lc}
\hline Biological function & List of genes \\
\hline Stem cell marker & OCT-4, hTERT \\
Thyroid differentiation marker & NIS \\
Thyroid transcription factor & PAX-8, TTF-1 \\
Invasion & MMP-2, MMP-9 \\
Cell cycle & p21 \\
Multifunctional transcription factor & c-MYC \\
\hline
\end{tabular}

to respond poorly to conventional chemotherapy $[18,19]$. Differentiation therapy, therapeutic intervention promoting differentiation rather than killing cancer cells, targets the tumor initiating stem cells and also possesses less toxicities. We focused on the clinically applicable HDAC inhibitor, VPA, as a way to induce redifferentiation of thyroid cancer stem-like or stem cells, because VPA is clinically available and has a well-documented side effects profile. To address the redifferentiation and aggressiveness reduction responsible for observed biological effects following VPA treatment, we monitored the responses of $8305 \mathrm{C}$ and B-CPAP cell lines, measuring different variables associated with the stem cells.
Proliferation assay results showed that VPA inhibits the growth of both cell lines, with IC50 of $3 \mathrm{mmol} / \mathrm{L}$ and $5 \mathrm{mmol} / \mathrm{L}$ for B-CPAP and $8305 \mathrm{C}$, respectively. The inhibition of cell proliferation was accompanied by the increased expression of the $p 21$ gene expression (Figures 4(a) and 4(b)). Catalano et al. showed that VPA induces cell-cycle arrest at G1/S by induction of $\mathrm{p} 21$ [20]. In our study, expression of $\mathrm{p} 21$ was increased dramatically in anaplastic cells and we believe that this might be p53-independent because p53 is mutated in B-CPAP [21] and 8305C [22] cell lines. In addition, the induction of $p 21$ in our study is in accordance with other study that VPA, as a class I HDAC inhibitor, activates the transcription of $p 21$ by increasing the acetylation of histone $\mathrm{H} 3$ and $\mathrm{H} 4$ [10]. Brzezinski et al. showed that with advancing tumor grade in thyroid carcinoma, the expression of the $\mathrm{p} 21$ is progressively lost [23].

Morphometric analysis, including $\mathrm{N}: \mathrm{C}$ ratio and cell sizes, has diagnostic and prognostic values by which pathologists evaluate histological grade. As a cell matures and becomes more differentiated, the size of its nucleus generally decreases. For example, "blast" forms of erythrocytes, leukocytes, and megakaryocytes start with an $\mathrm{N}: \mathrm{C}$ ratio of $4: 1$, which decreases to $2: 1$ or even $1: 1$ as they mature. Although the $\mathrm{N}$ : C ratio is fairly constant for a given cell type, 


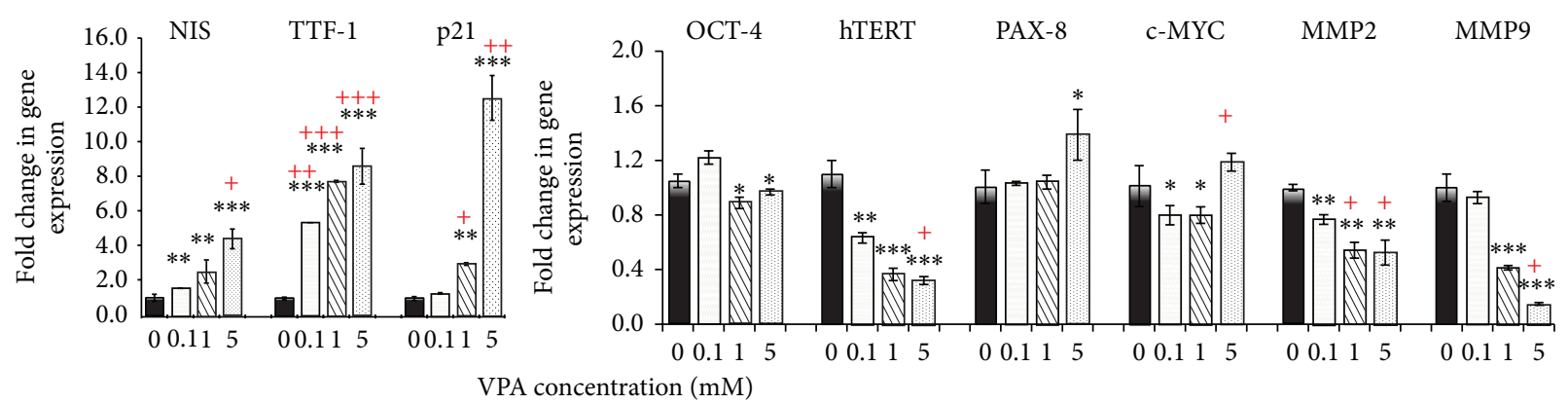

(a)

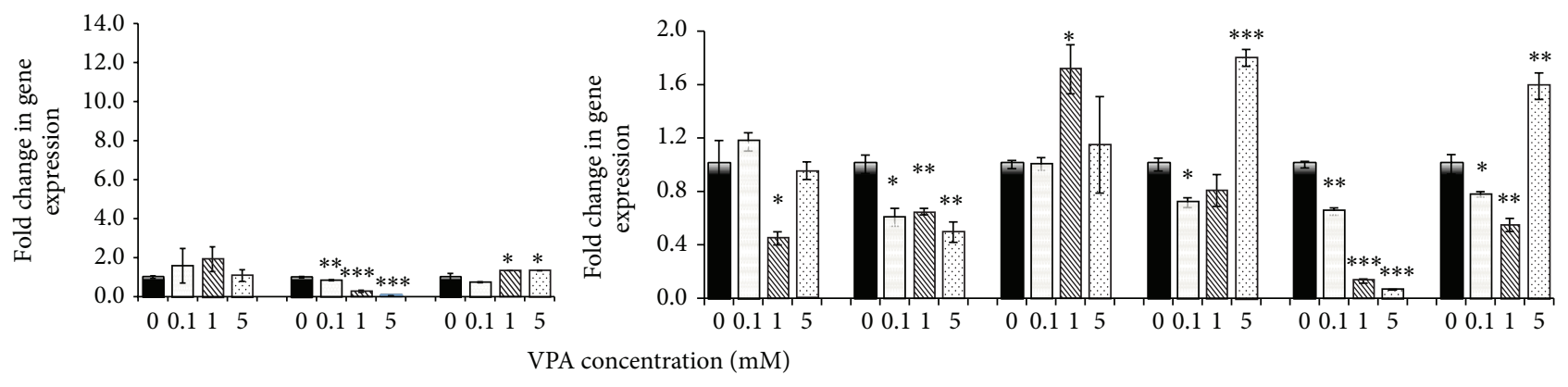

(b)

FIGURE 4: Effect of $96 \mathrm{~h}$ VPA treatment on mRNA transcriptional level. The relative mRNA expression of NIS, TTF1, p21, OCT-4, $h T E R T$, $P A X 8, c-M Y C, M M P 2$, and $M M P 9$ was measured following VPA treatment. The relative mRNA expression of each gene was measured by qRT-PCR in the $0.1,1$, and $5 \mathrm{mmol} / \mathrm{L}$ VPA treated $8305 \mathrm{C}$ (a) and B-CPAP (b) cell lines as described in Section 2. Data are presented as mean \pm SD. ${ }^{*} P<0.05,{ }^{* *} P<0.01$, and ${ }^{* * *} P<0.001 .+$ two cell lines compared to each other. Statistical significance was calculated using paired two-tailed Student's $t$-tests.

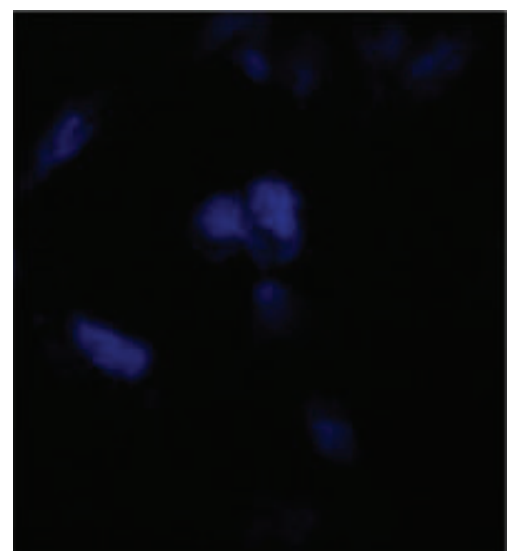

(a)

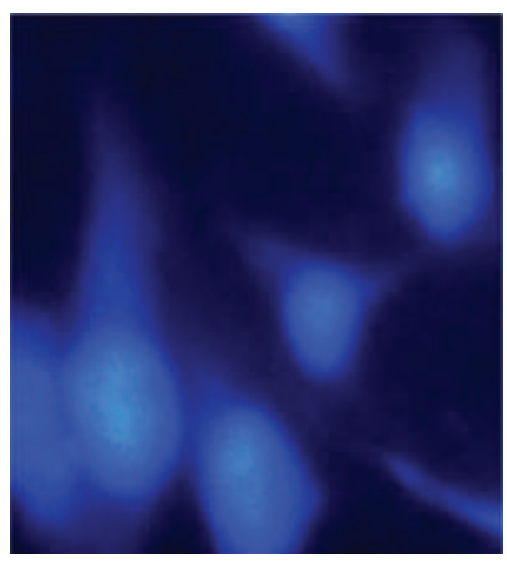

(b)

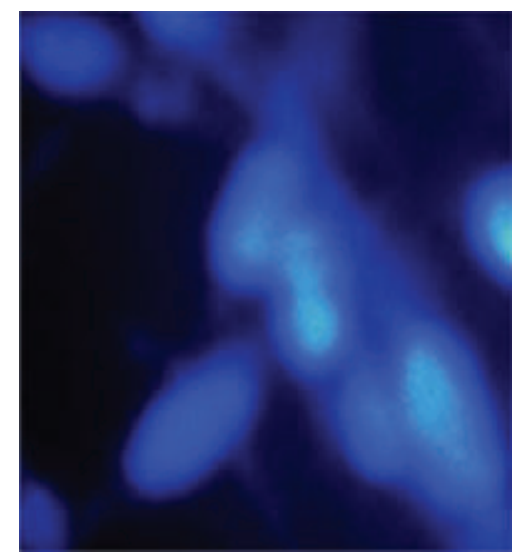

(c)

FIGURE 5: Hoechst 33342 staining of untreated control cells (a) versus $0.1 \mathrm{mmol} / \mathrm{L}$ (b) and $1 \mathrm{mmol} / \mathrm{L}$ (c) VPA treated 8305C cells. After exposure to VPA for $96 \mathrm{~h}$, fresh dilution of the Hoechst 33342 stock solution was added with the final concentration of $1 \mu \mathrm{g} / \mathrm{mL}$, incubated at $37^{\circ} \mathrm{C}$ for 15 minutes, protected from light. The stained cells were examined by IX70 fluorescence microscope using blue filter (excitation, $350 \mathrm{~nm}$; emission, $450 \mathrm{~nm}$ ) and photographed (magnification 40x).

an increased $\mathrm{N}: \mathrm{C}$ ratio is commonly associated with precancerous dysplasia as well as with malignant cells. Anaplastic thyroid cancer is undifferentiated (hence the name), categorized as grade IV with a large $\mathrm{N}$ : $\mathrm{C}$ ratio. Therefore, the $\mathrm{N}$ : C ratio could be exploited as an indicator to evaluate the differentiation status of the cells. According to the results of our study, the $\mathrm{N}$ : C ratio of the anaplastic cells reduced markedly after VPA exposure, accompanied by a morphological shift from a spindle shape to an epithelial-like shape in the treated cells. Giant cell and spindle shape cells were rarely observed following VPA treatment. The morphological changes following VPA treatment demonstrated by this study are further supported by the finding of a papillary-like shape of anaplastic thyroid cancer following combination therapy 
with VPA in a 51-year-old case by Noguchi et al. [24]. We observed that VPA was capable of restoring a differentiated phenotype.

Anaplastic thyroid carcinoma shows a marked epithelialmesenchymal transition (EMT) $[25,26]$, generating cell with stem-like characteristic. The loss of a high $\mathrm{N}: \mathrm{C}$ ratio and mesenchymal phenotype of the cells might suggest that stem cells have been committed to differentiate, losing the characteristic of stemness, which is further supported by downregulation of stem cell markers, as will be described later. The abrogation of EMT transition by HDAC inhibitor, trichostatin, has been shown in hepatocytes [27].

It has been shown that progenitor or stem cells which are enriched with $\mathrm{CD}_{133^{+}}$and OCT-4 have higher colonyforming capacity [5], in addition, the expression of OCT-4 in dedifferentiated cells leads to cancer stem-like cell features with the acquired ability to form tumor spheroids, increased resistance to chemotherapeutic agents, and increased tumorigenic capacity [28]. We evaluated the percentage of clonogenic cells to define subset cells endowed with colonyforming ability. The result of the colony-forming assay was correlated to the downregulation of OCT-4 expression by real-time PCR. The colony-formation assay is considered to be a stringent in vitro assay for malignantly transformed cells [17]. Therefore, VPA-induced differentiation could curtail the number of progenitor or stem cells.

The expression of hTERT in stem cells endows them with an indefinite cell proliferation, to bypass senescence and be immortal [29]. The stem cell marker OCT-4 and hTERT downregulation in this study supported the idea that VPA promoted differentiation in undifferentiated cells, which was confirmed further by colony formation and Hoechst 33342 staining. We observed that the expression of two known thyroid transcription factors, PAX8 and TTF1 and also NIS increased after VPA treatment in $8305 \mathrm{C}$ cells. Interestingly, however, TTF1 showed a reduction and PAX8 and NIS exhibited no clear pattern in B-CPAP cells. Downregulation of TTF1 and NIS expression in B-CPAP cells following HDAC inhibitors has been reported by Puppin et al. [30]. Our results being in line with those obtained by Fröhlich et al., which reported that VPA could not induce thyroid differentiation marker, NIS, in B-CPAP cell line [31]. The fact that redifferentiation with VPA seems capable of inducing the expression of the NIS gene is particularly intriguing because restoration of NIS activity could render less differentiated thyroid carcinomas amenable to radioiodine therapy. The result of our study gives further credence to the notion that anaplastic thyroid cancer might originate from cancer stem-like or stem cell which is supported by monitoring the expression pattern of the both cell lines after treatment. Expression pattern indicated that anaplastic cells, which show more stem cell related characteristics, followed an expected pattern by downregulation of stem cell markers, $h T E R T$ and $O C T-4$, and induction of thyroid differentiation markers, NIS, TTF1, and PAX8. In contrast, papillary cells showed no clear pattern relevant to re-differentiation following VPA treatment. VPA demonstrated some selectivity for stem-like anaplastic cells and less effect on differentiated papillary cells.
The side population cells that exclude Hoechst 33342 dye are enriched in stem cells and progenitor cells with relatively weak staining when cells were stained with Hoechst 33342. The result of our study shows that $8305 \mathrm{C}$ cells showed weak staining with Hoechst 33342 dye. In contrast, the VPA treated cells exhibited bright and homogenous staining compared to the control cells. This might imply that the stem cells have lost their ability to efflux Hoechst 33342 staining following redifferentiation induced by VPA. The result of Hoechst staining in our study is in accordance with another study that stem cells derived from human goiter, isolated as a side population by Hoechst 33342 efflux ability, displayed a high $\mathrm{N}: \mathrm{C}$ ratio and expressed the stem-cell marker OCT-4 [32].

Furthermore, it has been proposed that thyroid cancer stem cells might mediate metastasis, which remains the predominant cause of lethality in patients with thyroid cancer [17]. It is possible that approaches targeting stem cells could modulate invasive behavior of anaplastic thyroid carcinoma, a critical step in the clinical management of this disease. A growing amount of experimental evidence indicates that matrix metalloproteinases (MMPs) family members are involved in almost all metastatic steps [33]. The B-CPAP and 8305C cells have increased, compared to normal thyrocytes, the expression of MMP2 and MMP9, mediating the aggressive behavior in the both cell lines. Our study provides evidence supporting a role for VPA in suppressing aggressiveness through alteration of genes directly involved in metastasis. This could be achieved by transcriptional repression of key MMPs, MMP2 and MMP9.

Clinical trials for the use of VPA in the treatment of malignant tumors are already under way; however, its mechanism in anaplastic thyroid cancer, which might originate from thyroid cancer stem cells, is poorly understood. This study shows that VPA is effective on ATC and presumably reduction of aggressiveness and stem cell markers are the underlying mechanisms of VPA effect on anaplastic thyroid carcinoma, suggesting that reduction of aggressiveness and stemness may be a potential therapeutic target for undifferentiated and/or cancer stem cells. The biological behavior of cell lines does not always reproduce the biological behavior of the real tumor. Therefore, further in vivo and clinical studies, since the VPA is clinically applicable, are needed to determine the clinical efficacy of VPA on invariably lethal anaplastic thyroid carcinoma.

\section{Conflict of Interests}

The authors declare that there is no conflict of interests regarding the publication of this paper.

\section{Authors' Contribution}

Vahid Haghpanah and Mohsen Malehmir contributed equally to this work. 


\section{Acknowledgments}

This work was supported by Endocrinology and Metabolism Research Center (EMRC) and Iranian Stem Cell Network, Hematology-Oncology and Stem Cell Transplantation Research Center, Tehran University of Medical Sciences.

\section{References}

[1] C. Schmutzler and J. Koehrle, "Innovative strategies for the treatment of thyroid cancer," The European Journal of Endocrinology, vol. 143, no. 1, pp. 15-24, 2000.

[2] A. Vivaldi, F. Y. Miasaki, R. Ciampi et al., "Re-differentiation of thyroid carcinoma cell lines treated with 5-Aza-2'-deoxycytidine and retinoic acid," Molecular and Cellular Endocrinology, vol. 307, no. 1-2, pp. 142-148, 2009.

[3] D. Shibru, K. W. Chung, and E. Kebebew, "Recent developments in the clinical application of thyroid cancer biomarkers," Current Opinion in Oncology, vol. 20, no. 1, pp. 13-18, 2008.

[4] T. Takano and N. Amino, "Fetal cell carcinogenesis: a new hypothesis for better understanding of thyroid carcinoma," Thyroid, vol. 15, no. 5, pp. 432-438, 2005.

[5] G. Zito, P. Richiusa, A. Bommarito et al., "In vitro identification and characterization of CD133(pos) cancer stem-like cells in anaplastic thyroid carcinoma cell lines," PLoS ONE, vol. 3, no. 10, Article ID e3544, 2008.

[6] M. Derwahl, "Linking stem cells to thyroid cancer," Journal of Clinical Endocrinology and Metabolism, vol. 96, no. 3, pp. 610613, 2011.

[7] N. Mitsutake, A. Iwao, K. Nagai et al., "Characterization of side population in thyroid cancer cell lines: cancer stem-like cells are enriched partly but not exclusively," Endocrinology, vol. 148, no. 4, pp. 1797-1803, 2007.

[8] S. Sell, "Cancer stem cells and differentiation therapy," Tumor Biology, vol. 27, no. 2, pp. 59-70, 2006.

[9] J. E. Bolden, M. J. Peart, and R. W. Johnstone, "Anticancer activities of histone deacetylase inhibitors," Nature Reviews Drug Discovery, vol. 5, no. 9, pp. 769-784, 2006.

[10] S. Lee, J. R. Park, M. S. Seo et al., "Histone deacetylase inhibitors decrease proliferation potential and multilineage differentiation capability of human mesenchymal stem cells," Cell Proliferation, vol. 42, no. 6, pp. 711-720, 2009.

[11] S. R. Choudhury, S. Karmakar, N. L. Banik, and S. K. Ray, "Valproic acid induced differentiation and potentiated efficacy of taxol and nanotaxol for controlling growth of human glioblastoma LN18 and T98G Cells," Neurochemical Research, vol. 36, no. 12, pp. 2292-2305, 2011.

[12] C. P. Gan, S. Hamid, S. Y. Hor et al., "Valproic acid: growth inhibition of head and neck cancer by induction of terminal differentiation and senescence," Head and Neck, vol. 34, no. 3, pp. 344-353, 2012.

[13] S. Landreville, O. A. Agapova, K. A. Matatall et al., "Histone deacetylase inhibitors induce growth arrest and differentiation in uveal melanoma," Clinical Cancer Research, vol. 18, no. 2, pp. 408-416, 2012.

[14] M. Malehmir, V. Haghpanah, B. Larijani et al., "Multifaceted suppression of aggressive behavior of thyroid carcinoma by alltrans retinoic acid induced re-differentiation," Molecular and Cellular Endocrinology, vol. 348, no. 1, pp. 260-269, 2012.

[15] M. Todaro, F. Iovino, V. Eterno et al., "Tumorigenic and metastatic activity of human thyroid cancer stem cells," Cancer Research, vol. 70, no. 21, pp. 8874-8885, 2010.
[16] S. Friedman, M. Lu, A. Schultz, D. Thomas, and R. Y. Lin, "CD $133^{+}$anaplastic thyroid cancer cells initiate tumors in immunodeficient mice and are regulated by thyrotropin," PLoS ONE, vol. 4, no. 4, Article ID e5395, 2009.

[17] R. Y. Lin, “Thyroid cancer stem cells," Nature Reviews Endocrinology, vol. 7, no. 10, pp. 609-616, 2011.

[18] N. Fortunati, M. G. Catalano, K. Arena, E. Brignardello, A. Piovesan, and G. Boccuzzi, "Valproic acid induces the expression of the $\mathrm{Na}^{+} / \mathrm{I}^{-}$symporter and iodine uptake in poorly differentiated thyroid cancer cells," Journal of Clinical Endocrinology and Metabolism, vol. 89, no. 2, pp. 1006-1009, 2004.

[19] S. Chateauvieux, F. Morceau, M. Dicato, and M. Diederich, "Molecular and therapeutic potential and toxicity of valproic acid," Journal of Biomedicine and Biotechnology, vol. 2010, Article ID 479364, 18 pages, 2010.

[20] M. G. Catalano, N. Fortunati, M. Pugliese et al., "Valproic acid induces apoptosis and cell cycle arrest in poorly differentiated thyroid cancer cells," Journal of Clinical Endocrinology and Metabolism, vol. 90, no. 3, pp. 1383-1389, 2005.

[21] A. M. Meireles, A. Preto, A. S. Rocha et al., "Molecular and genotypic characterization of human thyroid follicular cell carcinoma-derived cell lines," Thyroid, vol. 17, no. 8, pp. 707-715, 2007.

[22] K. Ohnishi, I. Ota, A. Takahashi et al., "Glycerol as a chemical chaperone enhances radiation-induced apoptosis in anaplastic thyroid carcinoma cells," Molecular Cancer, vol. 1, article 4, 2002.

[23] J. Brzezinski, A. Migodzinski, A. Toczek, J. Tazbir, and M. Dedecjus, "Patterns of cyclin E, retinoblastoma protein, and p21Cip1/WAF1 immunostaining in the oncogenesis of papillary thyroid carcinoma," Clinical Cancer Research, vol. 11, no. 3, pp. 1037-1043, 2005.

[24] H. Noguchi, H. Yamashita, T. Murakami et al., "Successful treatment of anaplastic thyroid carcinoma with a combination of oral valproic acid, chemotherapy, radiation and surgery," Endocrine Journal, vol. 56, no. 2, pp. 245-249, 2009.

[25] J. Liu and R. E. Brown, "Immunohistochemical detection of epithelialmesenchymal transition associated with stemness phenotype in anaplastic thyroid carcinoma," International Journal of Clinical and Experimental Pathology, vol. 3, no. 8, pp. 755762, 2010.

[26] R. Y. Lin, "New insights into thyroid stem cells," Thyroid, vol. 17, no. 10, pp. 1019-1023, 2007.

[27] A. Kaimori, J. J. Potter, M. Choti, Z. Ding, E. Mezey, and A. A. Koteish, "Histone deacetylase inhibition suppresses the transforming growth factor betal-induced epithelial-tomesenchymal transition in hepatocytes," Hepatology, vol. 52, no. 3, pp. 1033-1045, 2010.

[28] S. M. Kumar, S. Liu, H. Lu et al., "Acquired cancer stem cell phenotypes through Oct4-mediated dedifferentiation," Oncogene, vol. 31, no. 47, pp. 4898-4911, 2012.

[29] P. Phatak and A. M. Burger, "Telomerase and its potential for therapeutic intervention," The British Journal of Pharmacology, vol. 152, no. 7, pp. 1003-1011, 2007.

[30] C. Puppin, F. d'Aurizio, A. V. d'Elia et al., "Effects of histone acetylation on sodium iodide symporter promoter and expression of thyroid-specific transcription factors," Endocrinology, vol. 146, no. 9, pp. 3967-3974, 2005.

[31] E. Fröhlich, P. Brossart, and R. Wahl, "Induction of iodide uptake in transformed thyrocytes: a compound screening in cell 
lines," The European Journal of Nuclear Medicine and Molecular Imaging, vol. 36, no. 5, pp. 780-790, 2009.

[32] L. Lan, D. Cui, K. Nowka, and M. Derwahl, "Stem cells derived from goiters in adults form spheres in response to intense growth stimulation and require thyrotropin for differentiation into thyrocytes," Journal of Clinical Endocrinology and Metabolism, vol. 92, no. 9, pp. 3681-3688, 2007.

[33] I. Stamenkovic, "Matrix metalloproteinases in tumor invasion and metastasis," Seminars in Cancer Biology, vol. 10, no. 6, pp. 415-433, 2000. 


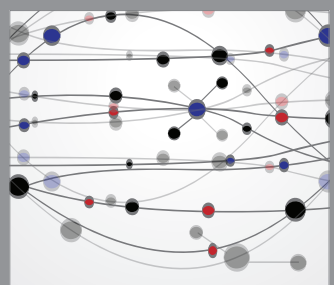

The Scientific World Journal
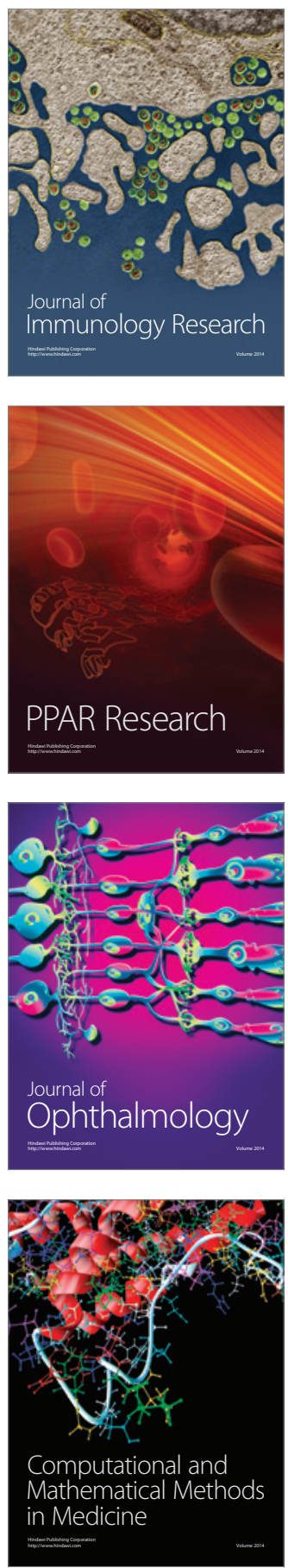

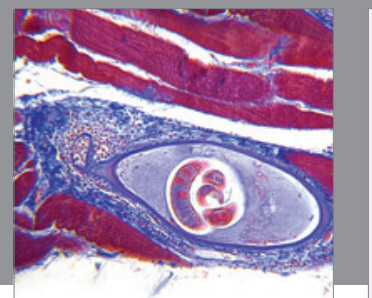

Gastroenterology

Research and Practice
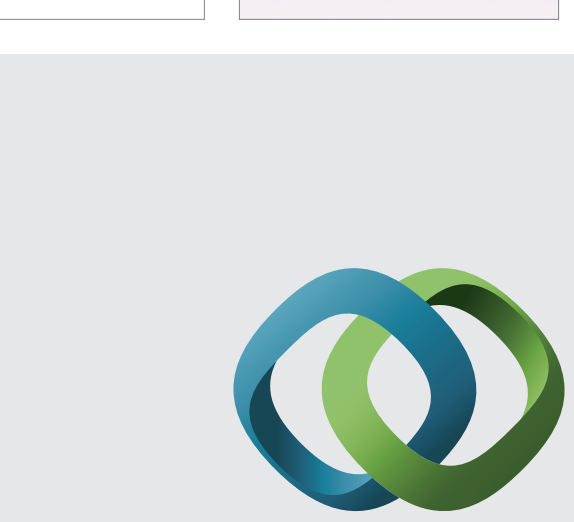

\section{Hindawi}

Submit your manuscripts at

http://www.hindawi.com
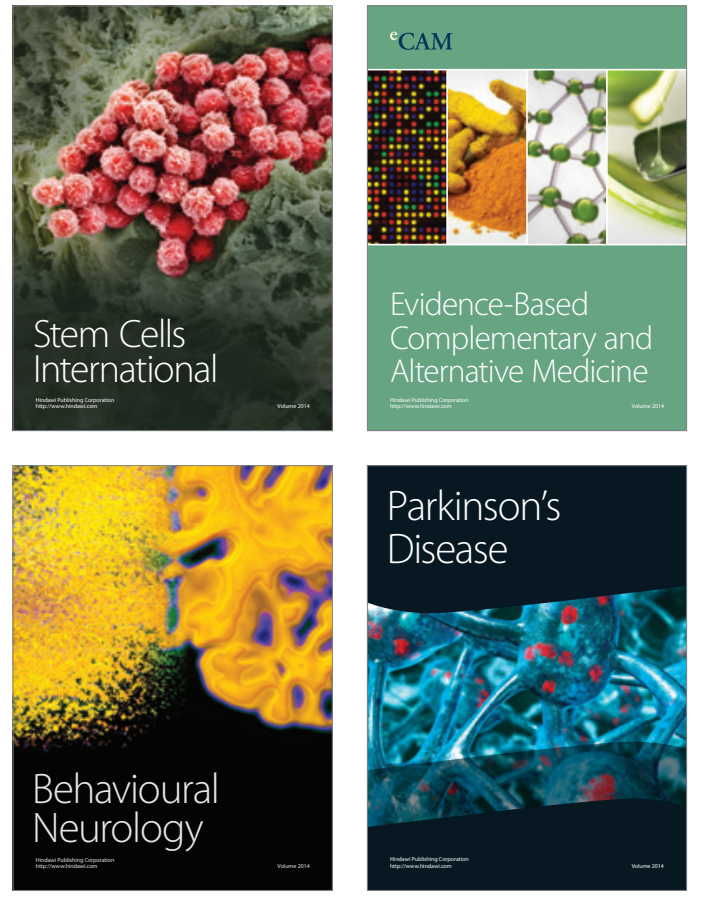
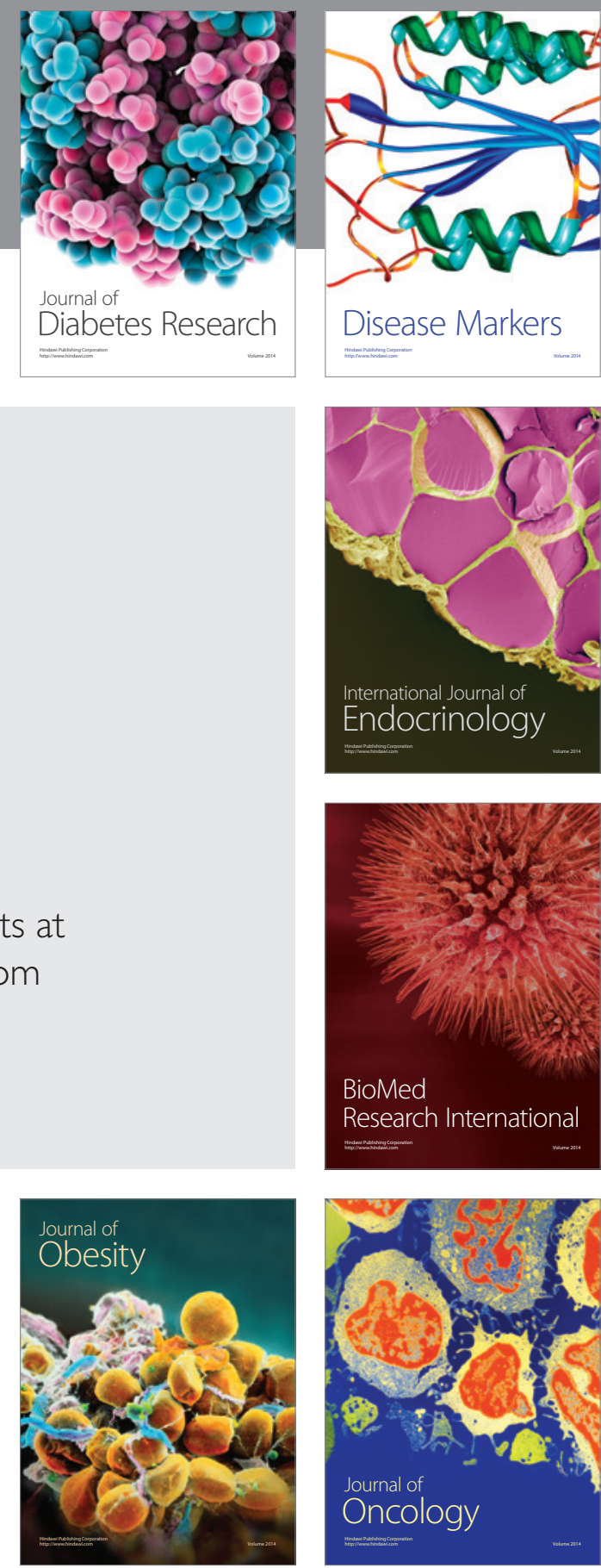

Disease Markers
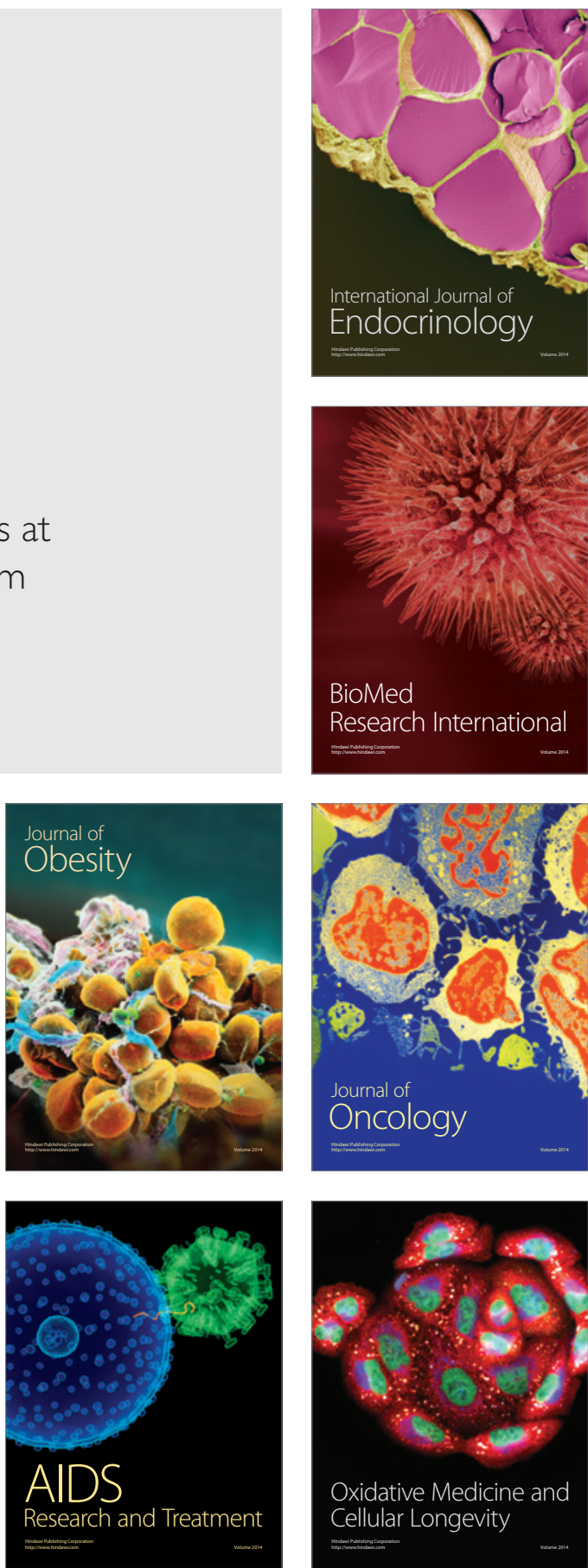